\title{
ENVOLVIMENTO E PERCEPÇÃO DOS CONSUMIDORES EM RELAÇÃO A MARCAS REPOSICIONADAS
}

\author{
Data de submissão: 06/05/2014 \\ Aceite: 21/06/2016 \\ Flavia Szylit ${ }^{1}$ \\ Giuliana Isabella ${ }^{2}$ \\ Lucia Salmonson Guimarães Barros ${ }^{3}$
}

\section{RESUMO}

O objetivo deste estudo foi identificar a relação entre o envolvimento do consumidor com uma marca, a percepção de seu reposicionamento e o consumo da marca reposicionada. Utilizou-se a rede de fast food Mc Donald's, que incluiu alimentos saudáveis em seu cardápio como parte do esforço de reposicionamento da marca. A coleta de dados foi feita por meio de questionários on-line, os quais foram respondidos por uma amostra de 214 indivíduos. Para a análise de dados, utilizou-se a econometria, permitindo a elaboração de um modelo de equações simultâneas com duas regressões, nas quais as variáveis dependentes são percepção e consumo. Os resultados indicaram que a percepção do reposicionamento depende positivamente da postura que o consumidor assume frente a uma alimentação balanceada e da frequência com que ele vai ao McDonald's. Essa relação é negativa para as variáveis idade, sexo, escolaridade do consumidor e da importância que ele dá ao valor nutricional dos alimentos. $O$ estudo sugere que a percepção é influenciada por estímulos pessoais e pelo envolvimento afetivo com a marca. Por outro lado, a aderência ao novo posicionamento não é influenciada por nenhum tipo de envolvimento, mas sim pelos estímulos pessoais dos consumidores.

Palavras Chaves: Envolvimento. Marcas Reposicionadas. Percepção de Marca. Alimentos., Econometria.

\footnotetext{
1 Possui mestrado em Administração de Empresas pela Fundação Getulio Vargas - SP (EAESP-FGV) e graduação em Administração pelo Insper - Instituto de Ensino e Pesquisa. E-mail: flavia.szylit@gmail.com

2 Professora de Dedicação Exclusiva do Insper - Instituto de Ensino e Pesquisa. Doutora pela Faculdade de Administração e Economia de Empresas da Universidade de São Paulo (FEA-USP) e Mestre em Administração de Empresas pela Fundação Getulio Vargas - SP (EAESPFGV). E-mail: giulianai@insper.edu.br.

3 Professora de Dedicação Exclusiva da Unifesp - Universidade Federal de São Paulo. Doutora em Administração de Empresas pela Escola Brasileira de Administração Pública e de Empresa da Fundação Getulio Vargas (EBAPE-FGV) e Mestre em Administração de Empresas pela Fundação Getulio Vargas - SP (EAESP-FGV). E-mail: lucia.barros@unifesp.br
} 


\section{INTRODUÇÃO}

A análise do envolvimento do consumidor em situações de compras ou relacionamento com produtos e marcas é um assunto recorrente na literatura (BRODERIK; MUELLER, 1999; CELSI; OLSON, 1998; FUCHS; DIAMANTOPOULOS, 2010) devido à sua importância ao estudo de marketing.

Um dos construtos abordados sobre esse envolvimento são os estímulos recebidos pelos consumidores, tanto do ambiente de compra, como de suas experiências prévias e características individuais, que acabam por impactar no comportamento de compra do consumidor (SANDHUSEN, 2000; SCHIFFMAN; KANUK, 2000). Outros estudos ainda consideram o relacionamento cognitivo, ligado às preferências pessoais do consumidor, incluindo o estilo de vida (Broderik; Mueller, 1999; Celsi; Olson, 1998; Souza, 2003) e o relacionamento afetivo com a marca a ser comprada (Bennett; Hartel; Mccoll-kennedy, 2005). Referente ao comportamento do consumidor, a percepção do posicionamento de marca muitas vezes é destacada como consequência dos estímulos e do envolvimento (Aaker; Shansby, 1982; FUCHS; DIAMANTOPOULOS, 2010).

Como muitas marcas são reposicionadas a fim de obter melhores resultados ou se adaptar a novas situações (Porter, 1996; Trout; Rivkin, 1996), é de se esperar que o comportamento do consumidor em relação a determinada marca mude juntamente com esse reposicionamento, se a nova imagem da marca for percebida.

Assim, o objetivo principal deste estudo é analisar se o envolvimento e os estímulos pessoais dos consumidores impactam na percepção do reposicionamento de uma marca, além de identificar se esses fatores, aliados à percepção, levam à aderência dessa mudança. Mais especificamente, foram propostas seis suposições que relacionam o estilo de vida do consumidor, o seu grau de envolvimento com uma marca e as suas características pessoais à percepção da mudança no posicionamento das marcas. As suposições estão descritas e detalhadas no decorrer do referencial teórico.

Para atingir esse objetivo, foi utilizado o contexto de redes de fast food, que por serem percebidas pelo consumidor como não saudáveis, começaram a incluir, na década passada, itens de maior valor nutritivo em seus cardápios (Boje; Carl, 2006), os quais se mantêm até hoje. Entretanto, ainda não se sabe se essas inclusões realmente promoveram alguma mudança no comportamento de compra ou na percepção do consumidor, mesmo sendo comuns artigos acadêmicos e matérias na mídia referentes aos prejuízos na saúde causados pelos produtos oferecidos por essas redes. Assim sendo, este artigo investiga também se as mudanças realizadas pelo Mc Donald's, a fim de mudar a sua imagem, realmente surtiram efeito no que tange à percepção do consumidor e seu comportamento de compra.

Galão, Crescitelli e Baccaro (2014) sugerem que se façam novos estudos relacionando o esforço de posicionamento de uma marca à percepção pelo consumidor, como também Ellickson, Misra e Nair (2012) apontam a falta de estudos empíricos sobre o reposicionamento. O presente estudo atende a essas sugestões e, assim, contribui para a literatura ao relacionar o reposicionamento de marca a uma mudança de percepção pelo consumidor e a sua compra. Além disso, investiga como características pessoais do consumidor impactam a percepção do reposicionamento e a compra e, finalmente, relaciona a percepção do reposicionamento ao comportamento de compra.

Analisar tal tema é relevante também para a prática do marketing, visto que várias empresas alimentícias buscam modificar a percepção do cliente por meio de uma mudança de cardápio, ou seja, tentam mudar um posicionamento de marca por meio da inclusão de serviços e produtos oferecidos. Este estudo servirá para esclarecer aos gestores das redes o que leva um indivíduo a perceber, ou não, e aderir, ou não, a essas mudanças, ajudando assim a determinar o foco de futuras campanhas de marketing. 
Este artigo está dividido da seguinte forma: apresentação do objetivo de pesquisa, seguido pela justificativa teórica, metodologia e análise econometria. Finaliza-se com uma discussão dos resultados e conclusão.

\section{REVISÃO DA LITERATURA}

A seguir, é apresentada uma revisão bibliográfica dos assuntos abordados neste trabaIho, juntamente com a apresentação de suposições.

\subsection{Posicionamento de Marca}

A escolha da estratégia de marca que maximize os resultados e o valor do cliente é um tema importante para gerentes de marketing (REGO; OLIVEIRA; LUCE, 2008). A construção da marca implica na escolha de um posicionamento adequado (MILLER, 2014), o que inclui definir a proposição de venda e a imagem da marca (Aaker; Shansby, 1982).

O posicionamento de uma marca diz respeito a como sua imagem é mostrada ao cliente (Yakimova; Beverland, 2005), muitas vezes relacionando-a a seus competidores (PAHARIA; AVERY; KEINAN, 2014). Ele indica aos consumidores o que a marca representa, o que ela é e como deveria ser percebida e avaliada (MONGA; GÜRHAN-CANLI, 2012), afetando diretamente as preferências dos consumidores, vendas e rendimentos (Giraldi; Spinelli; Merlo, 2003). No entanto, a escolha de um posicionamento único não é suficiente por si só para garantir vantagem competitiva sustentável (Porter, 1996) fazendo com que marcas sejam frequentemente reposicionadas (Schiffman; Kanuk, 2000; CHIABI; GONÇALVES, 2010; WANG; SHAVER, 2014).

Uma das estratégias de reposicionamento utilizadas é a de me too, que significa seguir o posicionamento adotado por uma marca líder (CARPENTER; NAKAMOTO, 1989; Trout; Rivkin, 1996). Oposta a ela está o reposicionamento radical, que significa realizar mudanças significativas na marca, no portfólio de produtos, nos formatos de loja etc. Outra estratégia é adotar uma atitude indecisa, ou seja, combinar os benefícios de uma posição de sucesso, mantendo a sua posição atual. Essa estratégia implica em trazer novas características, serviços ou tecnologias para as atividades que a empresa já realiza (Porter, 1996). Ainda outra é o incrementalismo, na qual a empresa faz mudanças incrementais no posicionamento de suas marcas em vez de uma mudança mais substancial (Martens; Matthyssens; Vandenbempt, 2012). O Quadro 1 resume essas estratégias de reposicionamento.

Quadro 1 - Estratégias de reposicionamento

\begin{tabular}{|c|l|c|}
\hline Estratégia & \multicolumn{1}{|c|}{ Conceito } & Referência \\
\hline Me Too & $\begin{array}{l}\text { Seguir o posicionamento adotado por } \\
\text { uma marca líder. }\end{array}$ & $\begin{array}{c}\text { CARPENT; NAKAMOTO, 1989; } \\
\text { TROUT; RIVKIN, 1996 }\end{array}$ \\
\hline Reposicionamento Radical & Realizar mudanças significativas. & CORSTJENS; DOYLE, 1989 \\
\hline Atitude Indecisa & $\begin{array}{l}\text { Trazer novas características, serviços } \\
\text { ou tecnologias para as atividades que } \\
\text { a empresa já realiza. }\end{array}$ & PORTER, 1996 \\
\hline Incrementalismo & $\begin{array}{l}\text { A empresa faz mudanças incrementais } \\
\text { no posicionamento de suas marcas, ao } \\
\text { invés de uma mudança mais substancial. }\end{array}$ & $\begin{array}{c}\text { MARTENS, MATTHYSSENS, VAN- } \\
\text { DENBEMPT, 2012 }\end{array}$ \\
\hline
\end{tabular}


Além disso, o posicionamento que uma marca deseja transmitir nem sempre é o percebido pelos consumidores (FRELING; CROSNO; HENARD, 2011). Logo, é relevante entender como a percepção dos consumidores é formada, levando em conta que a mente humana é limitada, avessa à confusão, insegura, difícil de modificar e suscetível à perda de foco (Trout; Rivkin, 1996).

\subsection{Envolvimento}

Um dos fatores que influencia a resposta em relação a uma marca é o grau de envolvimento, que pode levar a diferentes níveis de lealdade (Bennett; Hartel; Mccoll-Kennedy, 2005; Bowden; Dagger; Elliott, 2009), avaliação (PALMER, 2010) e satisfação do cliente (Oliver; Rust; Varki, 1997).

O envolvimento pode ser relacionado ao produto, à mensagem ou ao indivíduo que os percebe. No caso deste estudo, busca-se analisar o envolvimento com os produtos, com foco nos alimentícios, "cuja compra e consumo rotineiros são normalmente objeto de comportamentos que caracterizam baixo envolvimento, enquanto compras de alimentos realizadas para atender situações especiais como festas ou celebrações são qualificadas como de alto envolvimento" (Souza, 2003, p. 18). Um produto de baixo envolvimento consiste em algo comprado sem grande contemplação e que não envolve o ego nem muito dinheiro, sendo que uma má decisão de compra não implicaria em consequências muito negativas.

O envolvimento pode ser cognitivo e/ou afetivo: é chamado cognitivo quando focado no grau de relevância pessoal do conteúdo da marca baseado no seu valor utilitário; enquanto o envolvimento afetivo é o grau de relevância pessoal do conteúdo da marca baseado em apelos emocionais, ou seja, mais ligado à marca em si (ANDREWS; DURVASULA; AKHTER, 1990).

Segundo Broderik e Mueller (1999), o envolvimento cognitivo é um determinante primário do comportamento do consumidor, relacionando-o com diferentes aspectos do processo de consumo. Para Yi, Gong e Lee (2013), o envolvimento está relacionado ao ambiente onde o consumidor se encontra, assim como a sua motivação. De acordo com esses autores, a motivação, juntamente com um objetivo específico (procura por satisfação, por uma necessidade, por exemplo), pode influenciar o consumidor a buscar conhecer novos produtos ou serviços que satisfaçam a sua necessidade. As necessidades e o objetivo das pessoas estão relacionados às suas motivações, assim como ao envolvimento (Souza, 2003).

O envolvimento está relacionado com os produtos, com o grau de percepção da relevância, com a marca e com a propaganda (Celsi; Olson, 1998), "onde sons, cores e aromas são partes rotineiras dos seus diversos ambientes, representados por anúncios, embalagens de produtos, comerciais de rádio e televisão, outdoors, panfletos e similares" (Souza, 2003, p. 16).

Portanto, como a teoria sugere que o envolvimento cognitivo leva à diferença na percepção, faz sentido imaginar que

S1a: O grau de envolvimento de um indivíduo com hábitos saudáveis causa diferença na percepção da mudança de cardápio de redes fast food.

S1b: O grau de envolvimento de um indivíduo com hábitos saudáveis causa diferença no consumo dos novos alimentos no cardápio de redes de fast food.

Essas suposições serão testadas para cada um dos itens relacionados ao grau de envolvimento de um indivíduo com hábitos saudáveis. 


\subsection{Envolvimento com Marca}

As empresas, as organizações, os produtos, os serviços e até mesmo as pessoas, ao se relacionarem com os consumidores, fazem-no por meio de marcas. As marcas podem ser consideradas o uso de um termo, símbolo, sinal, desenho, com palavras ou nomes, que identifiquem a empresa, organização, produto ou serviço (Aaker; Shansby, 1982; Kotler, 1998). As marcas reproduzem elementos da empresa e, portanto, podem estar ligadas a aspectos objetivos (racionais) e subjetivos (emocionais). Segundo Aaker (1998), uma marca bem solidificada no mercado pode influenciar a decisão de compra.

Segundo Kotler (1998), o consumidor observa uma clara distinção entre as marcas quando buscam comprar produtos diferentes, embora o seu envolvimento com a marca possa ser considerado baixo. Um chocolate, por exemplo, é considerado um produto de baixo envolvimento, mas há inúmeras diferenças entre as marcas existentes no mercado e ele é reconhecido facilmente.

Desta forma, a percepção da imagem de uma marca pode estar ligada também ao envolvimento afetivo com ela. Propõe-se então que

S2a: O envolvimento de um indivíduo com a rede fast food causa diferença na percepção da mudança de cardápio de redes fast food.

S2b: O envolvimento de um indivíduo com a rede fast food causa diferença no consumo dos novos alimentos no cardápio de rede fast food.

\subsection{Estímulos Pessoais}

Além do envolvimento cognitivo e afetivo, os estímulos físicos e pessoais de cada consumidor também podem influenciar percepção e consumo.

É lógico entender que as diferenças individuais podem mudar a maneira como uma posição de marca é percebida, já que a percepção é resultante de um processo no qual os estímulos são selecionados, organizados e interpretados. Esses estímulos são físicos, obtidos por meio do ambiente, e pessoais, predispostos devido a experiências prévias, o que leva a uma visão única de mundo para cada indivíduo (Schiffman; Kanuk, 2000). Os estímulos pessoais são influenciados pelas características pessoais de cada indivíduo, como idade, sexo, renda, escolaridade e estado civil (Sandhusen, 2000). Assim, diferentes indivíduos poderão ter diferentes percepções de marca.

Este estudo analisa se os estímulos pessoais dos consumidores impactam a percepção do posicionamento de marca. Mais especificamente, busca-se testar se um indivíduo com dadas características tende a perceber diferenças no posicionamento de uma marca mais acuradamente. Ainda é verificado se essa percepção, tal como o envolvimento, leva à aderência do consumidor a esse novo posicionamento.

Logo, temos as seguintes suposições para cada uma das características pessoais medidas no estudo:

S3a: As características pessoais de um indivíduo causam diferença na percepção da mudança de cardápio de redes fast food.

S3b: As características pessoais de um indivíduo causam diferença no consumo dos novos alimentos no cardápio de redes fast food. 
Na próxima seção serão apresentadas a base de dados utilizada e o método econométrico aplicado para testar as suposições. Posteriormente, os resultados obtidos serão discutidos e as conclusões apresentadas. Com isso, será examinado o papel do envolvimento na percepção de tentativas de mudança de posicionamento de marcas.

\section{METODOLOGIA}

A seleção da amostra é não probabilística e foi realizada por meio de um questionário criado no Google docs, o qual foi encaminhado para a rede de contato dos autores. A coleta foi feita por meio do método "bola de neve", onde um participante repassa para o outro. Para calcular a amostra mínima necessária, definiu-se o poder do teste (Power $=1-\beta e r r o$ prob. $\mathrm{II}=$ $0,95)$ e o tamanho do efeito $\left(f^{2}\right)$ fraco $=0,10$, acima do recomendado por Cohen (1988) e Hair et al. (2009), sendo utilizado o número de 50 preditores. Com t crítico de 1.99, são recomendados 134 questionários completos. Ao todo, 219 questionários foram obtidos. Devido a informações incompletas, foi necessário reduzir a base de análise para 214.

Utilizou-se como objeto de estudo a rede de fast food Mcdonald's por três principais motivos: 1) trata-se de uma marca forte, portanto, pode-se assumir que os respondentes da pesquisa conhecem a marca, o seu portfólio de produtos e o seu posicionamento anterior. Isso é fundamental, uma vez que não é possível perceber uma mudança no posicionamento quando não se conhece o posicionamento anterior; 2 ) houve um forte esforço de mudança de posicionamento: de oferecer somente produtos altamente calóricos para oferecer uma grande variedade de produtos saudáveis. Este fator também é importante, pois só é possível haver uma mudança de percepção caso haja uma mudança real no mix de marketing da marca; e 3) não se trata de uma marca de nicho, o que reduz a possibilidade de apenas alguns respondentes conhecerem o posicionamento anterior e posterior.

Foram mensurados o consumo e as mudanças na percepção dos consumidores que estão (ou não) envolvidos com a sua saúde.

O questionário foi desenvolvido com base no apresentado por Alfassi et al. (2005). Para medir o envolvimento com a marca, foi solicitado que os respondentes apontassem com que frequência eles consumiam alimentos do McDonald's, dadas faixas pré-determinadas. Para medir consumo e percepção, foi fornecida a cada respondente duas listas contendo 30 alimentos (nem todos presentes no cardápio do McDonald's), incluindo 13 itens de maior valor nutricional, que foram divulgados pelo McDonald's durante campanhas publicitárias. As duas listas eram iguais, mas em uma o respondente deveria marcar o que sabia existir no cardápio, enquanto na outra apenas o que consumia. Dessa forma, foi montado um índice de percepção e outro de consumo, ambos variando de 1 a 13, que representam o número de alternativas saudáveis indicadas pelos respondentes.

A fim de coletar dados referentes às características pessoais, foi perguntado para cada respondente a idade, sexo, faixa de renda, estado civil e escolaridade. Ademais, responderam também perguntas sobre o estilo de vida, como quanto tempo diário o respondente dedica a assistir televisão, à prática de exercícios físicos e a cada refeição do dia. Também foram feitas perguntas a respeito da importância que cada respondente dava à refeição balanceada, ao valor nutricional dos alimentos e a exercícios físicos, utilizando uma escala Likert de 7 pontos. 


\section{RESULTADOS E ANÁLISES}

\subsection{Análise Descritiva}

A idade média dos respondentes foi de 31,36 anos com desvio padrão de 10,89 , sendo $64,49 \%$ mulheres, $65,89 \%$ solteiros e $30,84 \%$ casados. A renda média familiar dos respondentes foi bastante variada, com 12,62\% entre 1 e 3 salários mínimos, 16,82\% entre 4 e 7, 30,37\% entre 8 e $15,21,96 \%$ de 16 e 30, e 18,69\% acima de 31 salários mínimos. Portanto, tem-se que, dessa amostra, 40\% dos respondentes tem uma renda média acima de 16 salários mínimos. Quanto à escolaridade, $37,85 \%$ possuem superior completo e $38,32 \%$ possuem escolaridade de especialização ou superior.

Com relação ao número de horas diárias que o respondente assiste televisão, temos: $\mu=1,77 ; \sigma=1,70$; o tempo em horas semanais destinados a exercícios físicos é igual a $\mu=3,13 ; \sigma=$ 2,65 ; e os minutos destinados a cada refeição, em média, equivalem a $\mu=41,64 ; \sigma=19,25$. Como comentado anteriormente, essas perguntas serviram para caracterizar o estilo de vida de cada indivíduo da amostra.

Com relação ao envolvimento com a marca, foi solicitado que os respondentes apontassem com que frequência eles consumiam alimentos do McDonald's. Como resultado teve-se que 2,34\% frequentam de 1 a 3 vezes por semana, 17,29\% de 1 a 5 vezes por mês, 25,70\% de 6 a 12 vezes por ano, 3,38\% de 1 a 5 vezes por ano e 17,29\% menos de uma vez por ano.

Para medir consumo e percepção de produtos (que sabiam existir no cardápio), os participantes apenas assinalaram os produtos. As Figuras 1 e 2 mostram o histograma do valor do índice por quantidade de respondentes para percepção e consumo respectivamente.

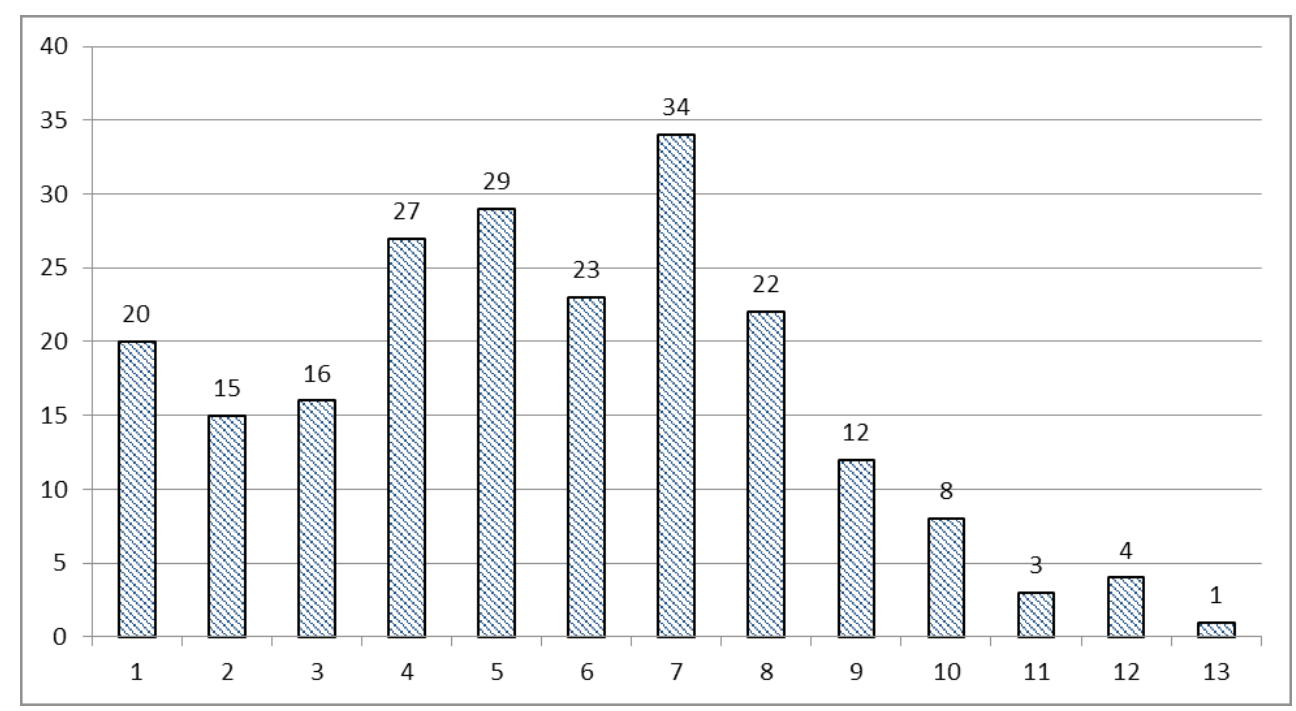

Figura 1. Histograma: Índice Percepção x Quantidade de respondentes Nota: $\mu=5,50 ; \sigma=2,80$ 


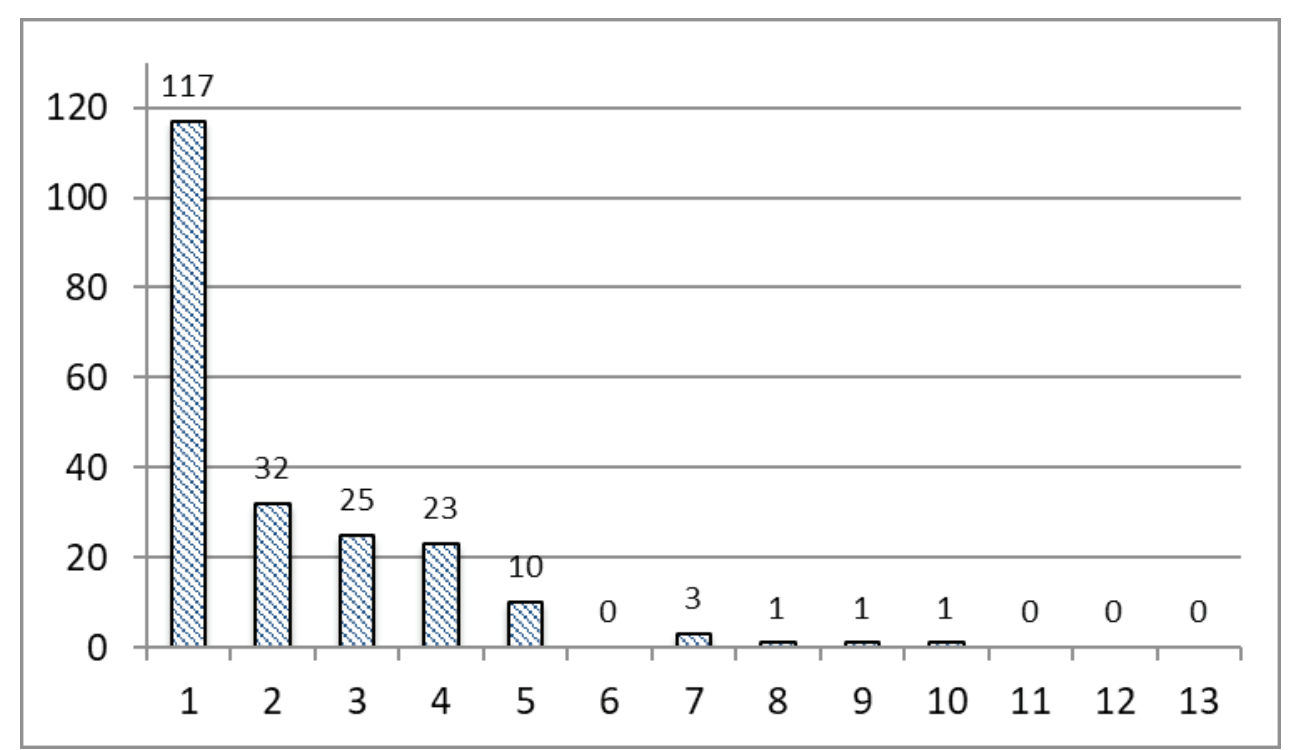

Figura 2. Histograma: Índice Consumo x Quantidade de respondentes Nota: $\mu=1,82 ; \sigma=1,84$

\subsection{Análise Econométrica}

A análise de dados foi feita por meio de equações simultâneas, já que nesse caso é preciso estimar uma regressão para percepção e outra para consumo. Ambas as equações possuem variáveis independentes iguais, mas o consumo depende da percepção. As estimativas foram feitas por meio de mínimos quadrados ordinais, que é a forma adequada quando a variável dependente é métrica, como é o caso de percepção e consumo (ANGRIST; PISCHKE, 2008; HAIR et al., 2009).

Algumas características, por serem qualitativas ou estarem em faixas, precisaram ser transformadas em dummy. Foi o caso de sexo, estado civil, renda, escolaridade, conhecimento do McDonald's e frequência de visitas ao McDonald's. Com exceção de sexo, todas as características possuíam mais de duas alternativas, portanto, para cada uma delas, foram criadas $n-1$ variáveis dummy, sendo " $n$ " o número de alternativas. A descrição dessas variáveis encontra-se na Tabela 1.

Tabela 1- Descrição das variáveis dummy

\begin{tabular}{lll}
\hline Característica & Variável & Descrição \\
\hline Sexo & Masculino & $\begin{array}{l}\text { Masculino }=1 \\
\text { Feminino }=0\end{array}$ \\
\hline Estado civil & Solteiro & $\begin{array}{l}\text { Solteiro }=1 \\
\text { Casado, Desquitado, e Viúvo }=0\end{array}$ \\
\cline { 2 - 3 } & Casado & $\begin{array}{l}\text { Casado }=1 \\
\text { Solteiro, Desquitado, e Viúvo }=0\end{array}$ \\
\cline { 2 - 3 } & Desquitado & Desquitado $=1$ \\
& Solteiro, Casado, e Viúvo $=0$ \\
\hline
\end{tabular}




\begin{tabular}{|c|c|c|}
\hline \multirow[t]{4}{*}{ Renda mensal familiar } & $\begin{array}{l}1 \text { a } 3 \text { salários } \\
\text { mínimos }\end{array}$ & $\begin{array}{l}1 \text { a } 3 \text { salários mínimos }=1 \\
4 \text { a } 7 \text { salários mínimos, } 8 \text { a } 15 \text { salários mínimos, } 16 \text { a } 30 \text { salá- } \\
\text { rios mínimos, e Mais que } 31 \text { salários mínimos }=0\end{array}$ \\
\hline & $\begin{array}{l}4 \text { a } 7 \text { salários } \\
\text { mínimos }\end{array}$ & $\begin{array}{l}4 \text { a } 7 \text { salários mínimos }=1 \\
1 \text { a } 3 \text { salários mínimos, } 8 \text { a } 15 \text { salários mínimos, } 16 \text { a } 30 \text { salá- } \\
\text { rios mínimos, e Mais que } 31 \text { salários mínimos }=0\end{array}$ \\
\hline & $\begin{array}{l}8 \text { a } 15 \text { salá- } \\
\text { rios mínimos }\end{array}$ & $\begin{array}{l}8 \text { a } 15 \text { salários mínimos }=1 \\
1 \text { a } 3 \text { salários mínimos, } 4 \text { a } 7 \text { salários mínimos, } 16 \text { a } 30 \text { salá- } \\
\text { rios mínimos, e Mais que } 31 \text { salários mínimos }=0\end{array}$ \\
\hline & $\begin{array}{l}16 \text { a } 30 \text { salá- } \\
\text { rios mínimos }\end{array}$ & $\begin{array}{l}16 \text { a } 30 \text { salários mínimos = } 1 \\
1 \text { a } 3 \text { salários mínimos, } 4 \text { a } 7 \text { salários mínimos, } 8 \text { a } 15 \text { salários } \\
\text { mínimos, e Mais que } 31 \text { salários mínimos }=0\end{array}$ \\
\hline \multirow[t]{5}{*}{ Escolaridade ${ }^{(1)}$} & $\begin{array}{l}\text { Fundamental } \\
\text { incompleto }\end{array}$ & $\begin{array}{l}\text { Fundamental incompleto = } 1 \\
\text { Médio incompleto, Médio completo, Superior incompleto, } \\
\text { Superior completo e Especialização, Pós-graduação, Mestra- } \\
\text { do ou Doutorado }=0\end{array}$ \\
\hline & $\begin{array}{l}\text { Médio in- } \\
\text { completo }\end{array}$ & $\begin{array}{l}\text { Médio incompleto = } 1 \\
\text { Fundamental incompleto, Médio completo, Superior incom- } \\
\text { pleto, Superior completo e Especialização, Pós-graduação, } \\
\text { Mestrado ou Doutorado }=0\end{array}$ \\
\hline & $\begin{array}{l}\text { Médio com- } \\
\text { pleto }\end{array}$ & $\begin{array}{l}\text { Médio completo = } 1 \\
\text { Fundamental incompleto, Médio incompleto, Superior in- } \\
\text { completo, Superior completo e Especialização, Pós-gradua- } \\
\text { ção, Mestrado ou Doutorado = } 0\end{array}$ \\
\hline & $\begin{array}{l}\text { Superior in- } \\
\text { completo }\end{array}$ & $\begin{array}{l}\text { Superior incompleto = } 1 \\
\text { Fundamental incompleto, Médio incompleto, Médio comple- } \\
\text { to, Superior completo e Especialização, Pós-graduação, Mes- } \\
\text { trado ou Doutorado }=0\end{array}$ \\
\hline & $\begin{array}{l}\text { Superior } \\
\text { completo }\end{array}$ & $\begin{array}{l}\text { Superior completo = } 1 \\
\text { Fundamental incompleto, Médio incompleto, Médio com- } \\
\text { pleto, Superior incompleto e Especialização, Pós-graduação, } \\
\text { Mestrado ou Doutorado }=0\end{array}$ \\
\hline \multirow[t]{4}{*}{ Frequência McDonald's } & $\begin{array}{l}1 \text { a } 3 \text { vezes } \\
\text { por semana }\end{array}$ & $\begin{array}{l}1 \text { a } 3 \text { vezes por semana }=1 \\
1 \text { a } 5 \text { vezes por mês, } 6 \text { a } 12 \text { vezes por ano, } 1 \text { a } 5 \text { vezes por } \\
\text { ano, e Menos de } 1 \text { vez por ano }=0\end{array}$ \\
\hline & $\begin{array}{l}1 \text { a } 5 \text { vezes } \\
\text { por mês }\end{array}$ & $\begin{array}{l}1 \text { a } 5 \text { vezes por mês }=1 \\
1 \text { a } 3 \text { vezes por semana, } 6 \text { a } 12 \text { vezes por ano, } 1 \text { a } 5 \text { vezes por } \\
\text { ano, e Menos de } 1 \text { vez por ano }=0\end{array}$ \\
\hline & $\begin{array}{l}6 \text { a } 12 \text { vezes } \\
\text { por ano }\end{array}$ & $\begin{array}{l}6 \text { a } 12 \text { vezes por ano }=1 \\
1 \text { a } 3 \text { vezes por semana, } 1 \text { a } 5 \text { vezes por mês, } 1 \text { a } 5 \text { vezes por } \\
\text { ano, e Menos de } 1 \text { vez por ano }=0\end{array}$ \\
\hline & $\begin{array}{l}1 \text { a } 5 \text { vezes } \\
\text { por ano }\end{array}$ & $\begin{array}{l}1 \text { a } 5 \text { vezes por ano }=1 \\
1 \text { a } 3 \text { vezes por semana, } 1 \text { a } 5 \text { vezes por mês, } 6 \text { a } 12 \text { vezes por } \\
\text { ano, e Menos de } 1 \text { vez por ano }=0\end{array}$ \\
\hline
\end{tabular}

A fim de garantir que as regressões não sofressem com problemas de multicolinearidade, foram calculadas as correlações entre as demais variáveis, como mostrado pela Tabela 2. Observa-se que Idade e Percepção têm uma correlação de -0,38; Importância de Balanceamento e Importância de Valor Nutricional de 0,41; e Percepção e Consumo de 0,52; enquanto as demais variáveis não têm correlação significativa. Devido a esses valores, acima de 0,3 será verificado o 
Fator de Inflação da Variância (VIF) após a estimação da regressão, para que seja verificado se existe algum problema de multicolinearidade caso essas variáveis sejam significativas.

Tabela 2 - Correlação entre variáveis

\begin{tabular}{|c|c|c|c|c|c|c|c|c|c|}
\hline & a & $b$ & c & $d$ & e & $f$ & g & $\mathrm{h}$ & $\mathrm{i}$ \\
\hline a. Horas de exercícios (semanais) & 1,00 & & & & & & & & \\
\hline b. Horas de TV (diárias) & $-0,04$ & 1,00 & & & & & & & \\
\hline c. Idade & 0,06 & 0,12 & 1,00 & & & & & & \\
\hline d. Importância de Balanceamento & 0,03 & 0,00 & 0,04 & 1,00 & & & & & \\
\hline e. Importância de Exercícios & 0,14 & 0,14 & 0,04 & 0,25 & 1,00 & & & & \\
\hline f. Importância de Valor Nutricional & 0,02 & 0,06 & 0,10 & 0,41 & 0,12 & 1,00 & & & \\
\hline g. Minutos destinados à refeição & 0,13 & $-0,03$ & 0,08 & 0,02 & $-0,02$ & $-0,04$ & 1,00 & & \\
\hline h. Percepção & $-0,02$ & $-0,09$ & $-0,38$ & 0,03 & $-0,07$ & $-0,23$ & 0,02 & 1,00 & \\
\hline i. Consumo & $-0,04$ & $-0,03$ & $-0,06$ & 0,10 & $-0,08$ & $-0,08$ & 0,12 & 0,52 & 1,00 \\
\hline
\end{tabular}

Dessa forma, foi estimado o seguinte sistema de equações simultâneas:

$$
\begin{aligned}
\text { Percepção }= & \beta_{0}+\beta_{1} \text { Idade }+\beta_{2} \text { Masculino }+\beta_{3} \text { Solteiro }+\beta_{4} \text { Casado }+\beta_{5} \text { Desquitado } \\
& +\beta_{6} 1 \mathrm{a} 3 \text { mínimos }+\beta_{7} 3 \mathrm{a} 7 \text { mínimos }+\beta_{8} 7 \mathrm{a} 15 \text { mínimos } \\
& +\beta_{9} 15 \mathrm{a} 30 \text { mínimos }+\beta_{10} \text { Fundamental incompleto } \\
& +\beta_{11} \text { Médio incompleto }+\beta_{12} \text { Médio completo }+\beta_{13} \text { Superior incompleto } \\
& +\beta_{14} \text { Superior completo }+\beta_{15} \text { Minutos destinados a refeição } \\
& +\beta_{16} \text { Horas de exercício (semanais) }+\beta_{17} \text { Horas de TV (diárias) } \\
& +\beta_{18} \text { Importância de Valor Nutricional } \\
& +\beta_{19} \text { Importância de Balancemaneto }+\beta_{20} \text { Importância de Exercícios } \\
& +\beta_{21} 1 \mathrm{a} 3 \text { por semana }+\beta_{22} 1 \mathrm{a} 5 \text { por mês }+\beta_{23} \text { Ga12 por ano } \\
& +\beta_{24} 1 \mathrm{a} 5 \text { por ano }
\end{aligned}
$$

$$
\begin{aligned}
\text { Consumo }= & \beta_{25} \\
& +\beta_{26} \text { Percepção }+\beta_{27} \text { Idade }+\beta_{28} \text { Masculino }+\beta_{29} \text { Solteiro }+\beta_{30} \text { Casado } \\
& +\beta_{34} \text { 7a15 mínimos }+\beta_{35} 15 \mathrm{a} 30 \text { mínimos }+\beta_{36} \text { Fundamental incompleto } \\
& +\beta_{37} \text { Médio incompleto }+\beta_{38} \text { Médio completo }+\beta_{39} \text { Superior incompleto } \\
& +\beta_{40} \text { Superior completo }+\beta_{41} \text { Minutos destinados a refeição } \\
& +\beta_{42} \text { Horas de exercício (semanais) }+\beta_{43} \text { Horas de TV (diárias) } \\
& +\beta_{44} \text { Importância de Valor Nutricional } \\
& +\beta_{45} \text { Importância de Balancemaneto }+\beta_{46} \text { Importância de Exercícios } \\
& +\beta_{47} 1 \mathrm{a} 3 \text { por semana }+\beta_{48} 1 \mathrm{a} 5 \text { por mês }+\beta_{49} \text { aa12 por ano } \\
& +\beta_{50} 1 \mathrm{a} 5 \text { por ano }
\end{aligned}
$$

As Tabelas 3 e 4 apresentam os resultados obtidos para cada uma das regressões propostas no sistema, percepção e consumo respectivamente. Pode-se observar que grande parte das variáveis para ambas as equações não são significativas, portanto, foram feitos ajustes em ambas as regressões a fim de retirar as variáveis não significativas. Chegou-se, assim, ao seguinte sistema: 


$$
\begin{aligned}
\text { Percepção }= & \beta_{0}+\beta_{1} \text { Idade }+\beta_{2} \text { Masculino }+\beta_{3} \text { Fundamental incompleto } \\
& +\beta_{4} \text { Importância de Valor Nutricional }+\beta_{5} \text { Importância de Balancema } \\
& +\beta_{c} 1 \mathrm{a} 3 \text { vor semana }+\beta_{-1} 1 \mathrm{a} 5 \text { vor mês }+\beta_{n} 6 \mathrm{a} 12 \text { vor ano }+\beta_{n} 1 \mathrm{a} 5 \text { vor }
\end{aligned}
$$

\section{Consumo $=\beta_{10}+\beta_{11}$ Percepção $+\beta_{12}$ Idade $+\beta_{13}$ Superior incompleto $+\beta_{14}$ Superior completo}

As variáveis consideradas são as que apresentaram resultado significativo com pelo menos $10 \%$ de significância. Observa-se que a variável "Desquitado" apresentou valor significante no primeiro sistema estimado, porém, no modelo final, passou a não ser mais significante ( $\mathrm{t}$ =

\begin{tabular}{|c|c|c|c|c|}
\hline Variável & Coeficiente & Erro padrão & $\mathrm{t}$ & Prob. \\
\hline$\beta_{0}$ & 10,03 & 2,96 & 3,39 & $0.00 * * *$ \\
\hline Idade & $-0,06$ & 0,02 & $-2,77$ & $0.01 * *$ \\
\hline Masculino & $-1,64$ & 0,37 & $-4,49$ & $0.00 * * *$ \\
\hline Solteiro & $-3,33$ & 2,44 & $-1,36$ & 0.18 \\
\hline Casado & $-3,57$ & 2,38 & $-1,50$ & 0.14 \\
\hline Desquitado & $-4,83$ & 2,55 & $-1,90$ & $0.06^{*}$ \\
\hline 1 a 3 salários mínimos & $-0,82$ & 0,73 & $-1,12$ & 0.27 \\
\hline 4 a 7 salários mínimos & $-0,35$ & 0,59 & $-0,58$ & 0.56 \\
\hline 8 a 15 salários mínimos & 0,02 & 0,51 & 0,04 & 0.97 \\
\hline 16 a 30 salários mínimos & $-0,50$ & 0,52 & $-0,95$ & 0.34 \\
\hline Fundamental incompleto & $-4,43$ & 2,43 & $-1,82$ & $0.07^{*}$ \\
\hline Médio incompleto & $-0,09$ & 1,75 & $-0,05$ & 0.96 \\
\hline Médio completo & 0,27 & 0,88 & 0,31 & 0.76 \\
\hline Superior incompleto & 0,25 & 0,51 & 0,49 & 0.62 \\
\hline Superior completo & 0,22 & 0,38 & 0,59 & 0.56 \\
\hline Minutos destinados à refeição & 0,01 & 0,01 & 0,68 & 0.50 \\
\hline Horas de exercícios (semanais) & 0,07 & 0,07 & 0,98 & 0.33 \\
\hline Horas de TV (diárias) & 0,01 & 0,10 & 0,05 & 0.96 \\
\hline Importância de Valor Nutricional & $-0,29$ & 0,11 & $-2,63$ & $0.01 * *$ \\
\hline Importância de Balanceamento & 0,24 & 0,12 & 2,01 & $0.05^{* *}$ \\
\hline Importância de Exercícios & $-0,15$ & 0,14 & $-1,05$ & 0.29 \\
\hline 1 a 3 vezes por semana & 3,92 & 1,17 & 3,36 & $0.00 * * *$ \\
\hline 1 a 5 vezes por mês & 3,08 & 0,60 & 5,16 & $0.00 * * *$ \\
\hline 6 a 12 vezes por ano & 2,63 & 0,52 & 5,06 & $0.00 * * *$ \\
\hline 1 a 5 vezes por ano & 1,72 & 0,49 & 3,50 & $0.00 * * *$ \\
\hline
\end{tabular}
$-1,39 ; p=0,17)$.

Tabela 3 - Resultado da regressão preliminar - Variável dependente: Percepção

Nota: $R^{2}=0,41 ; R^{2}$ ajustado $=0,34 ; F=5,53$ com Prob $(F)=0,00$

$* * *$ Valores altamente significativos $<0,00 ; * *$ valores muito significativos $<0,05$; valores significativos < 0,10 . 
Tabela 4 - Resultado da regressão preliminar - Variável dependente: Consumo

\begin{tabular}{|c|c|c|c|c|}
\hline Variável & Coeficiente & Erro padrão & $\mathrm{t}$ & Prob. \\
\hline$\beta_{25}$ & $-3,09$ & 2,12 & $-1,46$ & 0.15 \\
\hline Percepção & 0,37 & 0,05 & 7,41 & $0.00 * * *$ \\
\hline Idade & 0,03 & 0,02 & 2,06 & $0.04 * *$ \\
\hline Masculino & 0,04 & 0,27 & 0,14 & 0.89 \\
\hline Solteiro & 1,90 & 1,71 & 1,12 & 0.27 \\
\hline Casado & 1,57 & 1,66 & 0,94 & 0.35 \\
\hline Desquitado & 1,49 & 1,79 & 0,83 & 0.41 \\
\hline 1 a 3 salários mínimos & $-0,19$ & 0,51 & $-0,36$ & 0.72 \\
\hline 4 a 7 salários mínimos & $-0,02$ & 0,41 & $-0,04$ & 0.97 \\
\hline 8 a 15 salários mínimos & $-0,05$ & 0,35 & $-0,15$ & 0.88 \\
\hline 16 a 30 salários mínimos & 0,24 & 0,36 & 0,65 & 0.52 \\
\hline Fundamental incompleto & $-1,19$ & 1,70 & $-0,70$ & 0.48 \\
\hline Médio incompleto & $-1,14$ & 1,22 & $-0,94$ & 0.35 \\
\hline Médio completo & 0,12 & 0,61 & 0,20 & 0.84 \\
\hline Superior incompleto & $-0,67$ & 0,36 & $-1,88$ & $0.06^{*}$ \\
\hline Superior completo & $-0,39$ & 0,26 & $-1,50$ & 0.13 \\
\hline Minutos destinados à refeição & 0,01 & 0,01 & 1,16 & 0.25 \\
\hline Horas de exercícios (semanais) & $-0,05$ & 0,05 & $-1,03$ & 0.30 \\
\hline Horas de TV (diárias) & 0,02 & 0,07 & 0,22 & 0.83 \\
\hline Importância de Valor Nutricional & 0,02 & 0,08 & 0,31 & 0.75 \\
\hline Importância de Balanceamento & 0,10 & 0,08 & 1,16 & 0.25 \\
\hline Importância de Exercícios & $-0,11$ & 0,10 & $-1,14$ & 0.26 \\
\hline 1 a 3 vezes por semana & $-0,74$ & 0,84 & $-0,89$ & 0.38 \\
\hline 1 a 5 vezes por mês & 0,28 & 0,44 & 0,62 & 0.53 \\
\hline 6 a 12 vezes por ano & 0,30 & 0,38 & 0,79 & 0.43 \\
\hline 1 a 5 vezes por ano & 0,29 & 0,35 & 0,82 & 0.41 \\
\hline
\end{tabular}

Nota: $R^{2}=0,35 ; R^{2}$ ajustado = 0,26; $F=3,99 \operatorname{com} \operatorname{Prob}(F)=0,00$

$* * *$ Valores altamente significativos $<0,00 ; * *$ valores muito significativos $<0,05$; valores significativos < 0,10 .

A fim de evitar problemas de multicolinearidade, foram analisados os VIFs para ambas as regressões. Nenhuma das variáveis apresentou valores acima de 2,15 , logo, se concluiu que não existe multicolinearidade.

Também foi realizado o teste de White para heterocedasticidade, já que esse fenômeno estatístico é umas das suposições básicas do modelo de regressão linear. Para a regressão com Percepção como variável dependente (Regressão 1 ), a hipótese de homocedasticidade não foi rejeitada $(F=0,75 ; p=0,66)$. No entanto, para a regressão com Consumo como variável dependente (Regressão 2), essa hipótese foi rejeitada $(F=9,24 ; p<0,00)$. Sendo assim, foi necessário corrigir a regressão para heterocedasticidade.

Outra hipótese básica ainda é a normalidade dos erros, sendo relevante testar a hipótese nula de normalidade dos erros para ambas as regressões. Novamente a Regressão 1 não apresenta problemas $(p=0,37)$, porém rejeitamos a hipótese para a Regressão $2(p<0,00)$.

Assim, a partir das devidas correções chegou-se aos resultados apresentados nas Tabelas 5 e 6. 
Tabela 5 - Resultado da regressão - Variável dependente: Percepção

\begin{tabular}{ccccc}
\hline Variável & Coeficiente & Erro padrão & $\mathrm{t}$ & Prob. \\
\hline$\beta_{0}$ & 6,61 & 0,95 & 6,93 & 0,00 \\
Idade & $-0,07$ & 0,02 & $-4,31$ & 0,00 \\
Masculino & $-1,54$ & 0,33 & $-4,64$ & 0,00 \\
Fundamental incompleto & $-4,54$ & 2,33 & $-1,95$ & 0,05 \\
Importância de Valor Nutricional & $-0,31$ & 0,10 & $-2,98$ & 0,00 \\
Importância de Balanceamento & 0,19 & 0,11 & 1,69 & 0,09 \\
1 a 3 vezes por semana & 3,68 & 1,10 & 3,35 & 0,00 \\
1 a 5 vezes por mês & 2,96 & 0,58 & 5,14 & 0,00 \\
6 a 12 vezes por ano & 2,49 & 0,50 & 4,97 & 0,00 \\
1 a 5 vezes por ano & 1,64 & 0,47 & 3,51 & 0,00 \\
\hline
\end{tabular}

Nota: $R^{2}=0,38 ; R^{2}$ ajustado $=0,35 ; F=13,68$ com Prob $(F)=0,00$

Para a Regressão 1 , encontramos um $R^{2}$ de 0,38 , o que significa que as variáveis independentes do modelo explicam $38 \%$ da variação amostral da percepção. Já a estatística $F=13,68$ apresenta um p_value de 0,00, portanto, rejeitamos a hipótese nula do teste de igualdade dos parâmetros estimados. Assim, não existem variáveis redundantes nesse modelo.

Tabela 6 - Resultado da regressão - Variável dependente: Consumo

\begin{tabular}{ccccc}
\hline Variável & Coeficiente & Erro padrão & t & Prob. \\
\hline$\beta_{10}$ & $-0,79$ & 0,39 & $-2,04$ & 0.04 \\
Percepção & 0,39 & 0,05 & 7,78 & 0.00 \\
Idade & 0,02 & 0,01 & 2,73 & 0.01 \\
Superior incompleto & $-0,61$ & 0,34 & $-1,80$ & 0.07 \\
Superior completo & $-0,38$ & 0,23 & $-1,62$ & 0.11 \\
\hline
\end{tabular}

Nota: $R^{2}=0,30 ; R^{2}$ ajustado $=0,29 ; F=22,90$ com Prob $(F)=0,00$

Para a Regressão 2, encontramos um $R^{2}$ de 0,30 , o que significa que as variáveis independentes do modelo explicam $30 \%$ da variação amostral da percepção. A estatística $F(22,90)$ também apresenta um p_value de 0,00 , então rejeitamos a hipótese nula do teste de igualdade dos parâmetros estimados. Assim, da mesma forma que para a Regressão 1, não existem variáveis redundantes.

A fim de aprofundar a análise, foram estimados também os intervalos de confiança para os parâmetros de cada variável para as duas regressões, com nível de significância de $10 \%$, conforme vemos nas Tabelas 7 e 8.

Tabela 7 - Intervalo de confiança (90\%) - Variável dependente: Percepção

\begin{tabular}{cccc}
\hline Variável & Coeficiente & Abaixo & Acima \\
\hline$\beta_{0}$ & 6,61 & 5,04 & 8,19 \\
Idade & $-0,07$ & $-0,09$ & $-0,04$ \\
Masculino & $-1,54$ & $-2,09$ & $-0,99$ \\
Fundamental incompleto & $-4,54$ & $-8,39$ & $-0,69$ \\
Importância de Valor Nutricional & $-0,31$ & $-0,48$ & $-0,14$ \\
Importância de Balanceamento & 0,19 & 0,00 & 0,37 \\
1 a 3 vezes por semana & 3,68 & 1,87 & 5,50 \\
1 a 5 vezes por mês & 2,96 & 2,01 & 3,92 \\
6 a 12 vezes por ano & 2,49 & 1,66 & 3,31 \\
1 a 5 vezes por ano & 1,64 & 0,87 & 2,42 \\
\hline
\end{tabular}


Tabela 8 - Intervalo de confiança (90\%) - Variável dependente: Consumo

\begin{tabular}{cccc}
\hline Variável & Coeficiente & Abaixo & Acima \\
\hline$\beta_{10}$ & $-0,79$ & $-1,44$ & $-0,15$ \\
Percepção & 0,39 & 0,30 & 0,47 \\
Idade & 0,02 & 0,01 & 0,04 \\
Superior incompleto & $-0,61$ & $-1,17$ & $-0,05$ \\
Superior completo & $-0,38$ & $-0,76$ & 0,01 \\
\hline
\end{tabular}

Ainda mais, as regressões também foram estimadas para as variáveis padronizadas descritas nas Tabelas 9 e 10.

Tabela 9 - Variáveis padronizadas - Variável dependente: Percepção

\begin{tabular}{cccc}
\hline Variável & Coeficiente & $\begin{array}{c}\text { Coeficiente pa- } \\
\text { dronizado }\end{array}$ & $\begin{array}{c}\text { Elasticidade nas } \\
\text { médias }\end{array}$ \\
\hline$\beta_{0}$ Idade & 6,61 & 0,00 & 1,20 \\
Masculino & $-0,07$ & $-0,26$ & $-0,38$ \\
Fundamental incompleto & $-1,54$ & $-0,26$ & $-0,10$ \\
Importância de Valor Nutricional & $-4,54$ & $-0,11$ & 0,00 \\
Importância de Balanceamento & $-0,31$ & $-0,19$ & $-0,24$ \\
1 a 3 vezes por semana & 0,19 & 0,11 & 0,18 \\
1 a 5 vezes por mês & 3,68 & 0,20 & 0,02 \\
6 a 12 vezes por ano & 2,96 & 0,40 & 0,09 \\
1 a 5 vezes por ano & 2,49 & 0,39 & 0,12 \\
\hline
\end{tabular}

Tabela 10 - Variáveis padronizadas - Variável dependente: Consumo

\begin{tabular}{cccc}
\hline Variável & Coeficiente & $\begin{array}{c}\text { Coeficiente pa- } \\
\text { dronizado }\end{array}$ & $\begin{array}{c}\text { Elasticidade nas } \\
\text { médias }\end{array}$ \\
\hline$\beta_{10}$ & $-0,79$ & 0,00 & $-0,44$ \\
Percepção & 0,39 & 0,59 & 1,17 \\
Idade & 0,02 & 0,14 & 0,40 \\
Superior incompleto & $-0,61$ & $-0,12$ & $-0,05$ \\
Superior completo & $-0,38$ & $-0,10$ & $-0,08$ \\
\hline
\end{tabular}

\section{DISCUSSÃO DOS RESULTADOS}

A partir das Tabelas 5 e 6, apresentadas anteriormente com as regressões finais estimadas, podemos fazer a interpretação dos resultados. Primeiramente, quanto à Regressão 1, notamos que as características que influenciam a percepção são: Importância de Valor Nutricional, Importância de Balanceamento, Idade, Sexo, Escolaridade, e Frequência de visitas ao McDonald's.

Esses resultados estão de acordo com Freling, Crosno e Henard (2011), uma vez que não foram todos os consumidores que perceberam as mudanças de posicionamento do McDonalds. Os parâmetros de Importância de Valor Nutricional e Importância de Balanceamento são respectivamente $-0,31$ e 0,19 . Isso significa que uma pessoa que se importa com o valor nutricional dos alimentos percebe menos a mudança do cardápio do que alguém que não se preocupa. Por outro lado, uma pessoa que se importa com uma alimentação balanceada percebe mais a mudança do 
cardápio do que alguém que não se preocupa. Portanto, em termos da Suposição 1a, os efeitos são colidentes, pois uma variável de envolvimento tem efeito positivo enquanto outra tem efeito negativo, minimizando o efeito total do envolvimento com hábitos saudáveis. Logo, não é possível chegar a uma conclusão satisfatória a respeito dessa suposição.

A S2a - o envolvimento de um indivíduo com a rede fast food causa diferença na percepção da mudança de cardápio de redes fast food - foi suportada. Isso porque as variáveis de envolvimento com o objeto de estudo são significativas ao modelo. Os parâmetros para as variáveis dummy de frequência são: De 1 a 5 vezes ao ano: 1,64; De 6 a 12 vezes ao ano: 2,49; De 1 a 5 vezes por mês: 2,96; e De 1 a 3 vezes por semana: 3,68. Assim, quanto menos um indivíduo frequenta o McDonald's, menos ele percebe a mudança. Esse resultado está de acordo com Broderik e Mueller (1999), que explica que o envolvimento cognitivo influencia variáveis comportamentais.

Quanto aos parâmetros das características individuais, o parâmetro de idade é - $0,07,0$ que significa que indivíduos mais jovens tendem a perceber mais a mudança no cardápio; o parâmetro de Masculino é -1,54, demonstrando que mulheres tendem a perceber as mudanças mais que os homens; e Ensino Fundamental Incompleto tem o parâmetro de -4,54, o que significa que indivíduos que não completaram o ensino fundamental tendem a perceber menos a mudança do que aqueles que chegaram ao ensino médio, superior ou pós-graduação. Dessa forma, percebese que as características individuais e, portanto, os estímulos pessoais têm influência na percepção. Logo, a suposição S3a é suportada pelos resultados.

Pela Regressão 2, é possível observar que as variáveis que influenciam o consumo são: Percepção, Idade, Ensino Superior Incompleto e Ensino Superior Completo. O parâmetro da variável Percepção de 0,39 mostra o esperado, que uma maior percepção leva a um maior consumo. Como nenhuma das demais variáveis diz respeito nem a estilo de vida e nem a envolvimento com o McDonald's, rejeitamos tanto a S1b como a S2b.

Comprovamos a S3b uma vez que os estímulos pessoais têm influência no consumo, como comentado por Sandhusen (2000). O parâmetro para Idade de -0,02, apesar de pouco significativo, mostra que pessoas mais jovens tendem a consumir mais os novos alimentos do cardápio do que mais velhos. Os parâmetros para as dummy ligadas a educação são Superior Incompleto -0,61 e Superior Completo -0,38, o que indica que pessoas que ainda estão cursando uma faculdade, ou que já a concluíram, mas não fizeram especialização ou pós-graduação, tendem a consumir menos esses alimentos do que os demais.

Os resultados deste estudo sugerem que tanto o envolvimento quanto os estímulos pessoais influenciam na percepção da mudança de posicionamento de uma marca, estando em acordo com Schiffman e Kanuk (2000), que afirmam que estímulos do ambiente pessoal, assim como predisposição devido a experiências prévias, fornece uma visão individualizada aos consumidores. Quer dizer, consumidores diferentes percebem uma mesma marca de formas distintas, independentemente do esforço realizado para se transmitir uma imagem única, trazendo um desafio extra para gestores de marcas. O relacionamento encontrado entre envolvimento e percepção está de acordo com Bloch (1982), que comenta que, quando os consumidores têm maior interesse pelos produtos, eles buscam mais informação e prestam mais atenção às mensagens, o que poderia levar ao aumento da percepção de uma mudança no posicionamento da marca.

Por fim, os resultados sugerem que a percepção do reposicionamento de uma marca influencia positivamente no seu consumo. Isso suporta a importância de um reposicionamento adequado, quando for necessário, para o aumento do consumo de uma marca. 


\section{CONSIDERAÇÕES FINAIS}

As seis suposições testadas nesse artigo foram: 1) S1a: O grau de envolvimento de um indivíduo com hábitos saudáveis causa diferença na percepção da mudança de cardápio das redes fast food; 2) S1b: O grau de envolvimento de um indivíduo com hábitos saudáveis causa diferença no consumo dos novos alimentos no cardápio de redes de fast food; 3) S2a: O envolvimento de um indivíduo com a rede fast food causa diferença na percepção da mudança de cardápio de redes fast food; 4) S2b: O envolvimento de um indivíduo com o McDonald's causa diferença no consumo dos novos alimentos no cardápio de rede fast food; 5) S3a: As características pessoais de um indivíduo causam diferença na percepção da mudança de cardápio de redes fast food; e 6) S3b: As características pessoais de um indivíduo causam diferença no consumo dos novos alimentos no cardápio de redes fast food.

Dessas hipóteses conclui-se que S1a, S1b e S2b não são suportadas com base em análises econométricas, enquanto que S2a, S3a e S3b são suportadas. O Quadro 2 resume os resultados encontrados.

Quadro 2-Resumo dos resultados

\begin{tabular}{|l|c|}
\hline \multicolumn{1}{|c|}{ Suposições } & Resultados \\
\hline $\begin{array}{l}\text { S1a: O grau de envolvimento de um indivíduo com hábitos saudáveis cau- } \\
\text { sa diferença na percepção da mudança de cardápio de redes fast food. }\end{array}$ & Rejeitada pelos resultados. \\
\hline $\begin{array}{l}\text { S1b: O grau de envolvimento de um indivíduo com hábitos saudáveis causa } \\
\text { diferença no consumo dos novos alimentos no cardápio de redes fast food. }\end{array}$ & Rejeitada pelos resultados. \\
\hline $\begin{array}{l}\text { S2a: O envolvimento de um indivíduo com a rede fast food causa diferen- } \\
\text { ça na percepção da mudança de cardápio de redes fast food. }\end{array}$ & Suportada pelos resultados. \\
\hline $\begin{array}{l}\text { S2b: O envolvimento de um indivíduo com a rede fast food causa diferen- } \\
\text { ça no consumo dos novos alimentos no cardápio de rede fast food. }\end{array}$ & Rejeitada pelos resultados. \\
\hline $\begin{array}{l}\text { S3a: As características pessoais de um indivíduo causam diferença na per- } \\
\text { cepção da mudança de cardápio de redes fast food. }\end{array}$ & Suportada pelos resultados. \\
\hline $\begin{array}{l}\text { S3b: As características pessoais de um indivíduo causam diferença no con- } \\
\text { sumo dos novos alimentos no cardápio de redes fast food. }\end{array}$ & Suportada pelos resultados. \\
\hline
\end{tabular}

Os resultados sugerem que nem todos os consumidores percebem mudanças de posicionamento de uma marca, o que suporta os achados de Freling, Crosno e Henard (2011). Especificamente, os dados mostram que o grau de envolvimento dos indivíduos com a necessidade e o objetivo de adquirir produtos saudáveis não permitem um melhor reconhecimento quanto à variedade de produtos apresentados pelas empresas, mesmo ao considerarmos empresas de fast food, vistas atualmente como pouco saudáveis.

Os resultados mostram também que as características pessoais, no entanto, estão relacionadas à percepção de mudanças nos cardápios. Por fim, foi constatado que o envolvimento do indivíduo com a marca está relacionado à percepção de mudança de cardápio da rede, mas não ao consumo dos novos produtos. Percebe-se que os resultados corroboram grande parte da teoria, concluindo que estímulos pessoais e envolvimento afetivo influenciam a percepção dos consumidores quanto a uma determinada marca. No entanto, apenas estímulos pessoais levam a aderência dessa nova imagem (no caso do estudo, consumo dos novos itens do cardápio).

Essas conclusões são úteis para gestores de marketing, que devem levar em consideração, então, o envolvimento afetivo dos consumidores na hora de direcionar campanhas de marketing a fim de reposicionar alguma marca. Ao saber que o envolvimento está fortemente relacionado com a percepção de mudança, as marcas devem buscar estar próximas de seus consumidores para mais facilmente se comunicarem com eles. 
No caso das empresas que buscam incluir uma imagem de alimentos saudáveis, é necessário levar em consideração que as pessoas envolvidas em buscar uma melhor alimentação não necessariamente irão perceber mais essas mudanças. Ainda mais, diferentes níveis de envolvimento, por gerarem diferentes percepções, exigem diferentes estratégias de reposicionamento e comunicação (ASSAEL, 1998).

Este estudo, no entanto, possui algumas limitações. A amostra não foi controlada por variáveis, sendo coletada por conveniência. Assim, as variáveis demográficas como sexo, renda, idade ou escolaridade não possuíam distribuições homogêneas. Dessa forma, o resultado da análise pode ter sido influenciado. Ademais, o presente estudo utilizou apenas uma marca, considerada fortemente conhecida no mercado. Estudos futuros poderiam endereçar essas limitações.

Outra limitação foi o fato de haver um corte temporal, uma vez que a coleta de dados foi feita em apenas um período, após o reposicionamento. Como sugestão para esclarecer tal questão, seria interessante realizar estudos longitudinais. Por exemplo, a percepção e a compra da marca poderiam ser mensuradas antes e depois do reposicionamento, permitindo uma comparação. Outra sugestão seria mensurar a mudança na percepção e na compra ao longo do tempo.

Outras variáveis relacionadas a envolvimento com hábitos saudáveis podem ser testadas. Identificar diferenças na relação entre marcas de alto e baixo envolvimento pode contribuir para explorar o tema. Sugere-se ainda a utilização de outros tipos de empresas, tanto da área alimentícia como de outras áreas de consumo.

É possível, também, analisar se tipos diferentes de personalidade podem influenciar no envolvimento com a marca, a percepção e as atitudes. Além disso, cabe investigar se a influência de outras pessoas, como em redes sociais virtuais (ABBADE; FLORA; NORO, 2014), pode alterar o envolvimento com a marca, assim como os comportamentos relacionados a ela. Produtos de alto envolvimento também seriam bons candidatos a tal teste, comparando-se assim as empresas de serviços.

\section{REFERÊNCIAS}

Aaker, D. A. Marcas: Brand Equity gerenciando o valor da marca. São Paulo: Elsevier Brasil, 1998.

Aaker, D. A.; Shansby, J. G. Positioning your product. Business Horizons v. 25, n.3, p. 56-63, 1982.

ABBADE, E. B.; FLORA, A. D.; NORO, G. B. A influência interpessoal em redes sociais virtuais e as decisões de consumo. Revista de Administração da UFSM, v. 7, n.2, p. 265-278, 2014.

Alfassi, E. et al.. McDonald's com cardápio saudável? VIII SEMEAD. São Paulo, 2005.

ANDREWS, J. C.; DURVASULA, S.; AKHTER, S. H. A framework for conceptualizing and measuring the involvement construct in advertising research. Journal of advertising, v. 19, n. 4, p. 27-40, 1990.

ANGRIST, J. D.; PISCHKE, J-S. Mostly harmless econometrics: An empiricist's companion. Princeton University Press, 2008.

ASSAEL, H. Consumer behavior and marketing action. 6. ed. Cincinnati, Ohio: South-Western College Publishing, 1998.

Bennett, R.; Hartel, C.; McColl-Kennedy, J. Experience as a Moderator of Involvement and 
Satisfaction on Brand Loyalty in a Business-to-Business Setting. Industrial Marketing Management, v. 34, n. 1, p. 97-107. 2005.

BLOCH, P. H. Involvement beyond the purchase process: conceptual issues and empirical investigation. Advances in Consumer Research, v. 8, p. 61-65, 1982.

Boje, D. M.; Carl, R. The leadership of Ronald McDonald: Double narration and stylistic lines of transformation. The Leadership Quarterly, v.17; n. 1, p. 94-103, 2006.

Bowden, J. L.; Dagger, T. S.; Elliott, G. The role of relational antecedents and the effect of experience in the development of service loyalty. Australian and New Zealand Marketing Academy (ANZMAC) Conference. Melbourne. 2009.

Broderik, A. J.; Mueller, R. D. A theoretical and empirical exegesis of the consumer involvement construct: The psychology of the food shopper. Journal of Marketing Theory and Practice, v. 7, n. 4, p. 97-108, 1999.

CARPENTER, G. S.; NAKAMOTO, K. Consumer preference formation and pioneering advantage. Journal of Marketing Research, p. 285-298, 1989.

Celsi, R. L.; Olson, J. C. The role of involvement in attention and comprehension processes. The Journal of Consumer Research. v. 15, n. 2, p. 210-224, 1998.

CHIABI, A. L. G.; GONÇALVES, C. A. Análise de estratégia de mudança da marca acrescentando valor: um estudo da Vale. Revista de Administração da UFSM, v. 3, n.1, p. 131-143, 2010.

COHEN, J. Statistical power analysis for the behavioral sciences. Mahwah, NJ: Lawrence Erlbaum, 1988.

CORSTJENS, M.; DOYLE, P. Evaluating alternative retail repositioning strategies. Marketing Science, v. 8, n. 2, p. 170-180, 1989.

ELLICKSON, P. B.; MISRA, S.; NAIR, H. S. Repositioning dynamics and pricing strategy. Journal of Marketing Research, v. 49, n. 6, p. 750-772, 2012.

FRELING, T. H.; CROSNO, J. L.; HENARD, D. H. Brand personality appeal: conceptualization and empirical validation. Journal of the Academy of Marketing Science, v. 39, n. 3, p. 392-406, 2011.

FUCHS, C.; DIAMANTOPOULOS, A. Evaluating the effectiveness of brand-positioning strategies from a consumer perspective. European Journal of Marketing, v. 44, n. 11/12, p. 1763-1786, 2010.

GALÃO, F. P.; CRESCITELLI, E.; BACCARO, T. A. Comunicação Integrada de Marketing: Uma Ferramenta do Posicionamento Estratégico? UNOPAR Científica Ciências Jurídicas e Empresariais, v. 12, n. 01, 2014.

Giraldi, J. M., Spinelli, P. B., \& Merlo, E. Retail Store Image: Analysis of the implications for Store Positioning. REAd - Revista Eletrônica de Administração v.36, n.9, p. 203-222, 2003.

HAIR, J. F. et al. Análise multivariada de dados. Bookman, 2009.

KOTLER, P. Administração de marketing. $5^{\circ}$ ed. São Paulo: Atlas, 1998. 
MARTENS, R.; MATTHYSSENS, P.; VANDENBEMPT, K. Market strategy renewal as a dynamic incremental process. Journal of Business Research, v. 65, n. 6, p. 720-728, 2012.

MILLER, D. Brand-building and the elements of success: discoveries using historical analyses. Qualitative Market Research: An International Journal, v. 17, n. 2, p. 92-111, 2014.

MONGA, A.; GÜRHAN-CANLI, Z. The influence of mating mind-sets on brand extension evaluation. Journal of Marketing Research, v. 49, n. 4, p. 581-593, 2012.

OLIVER, R. L.; RUST, R. T.; VARKI, S. Customer delight: foundations, findings, and managerial insight. Journal of Retailing, v. 73, n. 3, p. 311-336, 1997.

PAHARIA, N.; AVERY, J.; KEINAN, A. Positioning Brands Against Large Competitors to Increase Sales. Journal of Marketing Research, v. 51, n. 6, p. 647-656, 2014.

PALMER, A. Customer experience management: a critical review of an emerging idea. Journal of Services Marketing, v. 24, n. 3, p. 196-208, 2010.

Porter, M. What is Strategy. Harvard Business Review, November, 61-78, 1996.

REGO, B. B.; OLIVEIRA, M. O. R.; LUCE, F. B. Uma discussão teórica da relação do valor da marca e do valor do cliente. Revista de Administração da UFSM, v. 1, n.2, p. 275-290, 2008.

Sandhusen, R. L. Marketing. New York: Barron's Educational Series, 2000.

Schiffman, L. G., \& Kanuk, L. L. Consumer Behavior. New Jersey: Prentice-Hall, 2000.

Souza, A. R. Hábitos de Consumo Alimentar e Envolvimento do Consumidor: um estudo na cidade do Recife- PE. Universidade Federal de Pernambuco: Tese de Mestrado, 2003.

Trout, J., \& Rivkin, S. The New Positioning. New York: McGraw-Hill, 1996.

WANG, R. D.; SHAVER, J. M. Competition-driven repositioning. Strategic Management Journal, v. 35, n. 11, p. 1585-1604, 2014.

Yakimova, R., \& Beverland, M. The brand-supportive firm: An exploration of organizational drivers of brand updating. Journal of Brand Management, v.12, n.6, p. 445-460, 2005.

YI, Y.; GONG, T.; LEE, H. The impact of other customers on customer citizenship behavior. Psychology \& Marketing, v. 30, n. 4, p. 341-356, 2013. 